\title{
Microscopic and flow cytometric semen assessment of Dutch AI-bucks: Effect of semen processing procedures and their correlation to fertility
}

\author{
K. Peterson ${ }^{a}$, M.A. P.M. Kappen ${ }^{b}$, P.J.F. Ursem ${ }^{a}$, J.O. Nöthling ${ }^{c}$, B. Colenbrander ${ }^{a}$ \\ and B.M. Gadella ${ }^{\text {a,d }}$ \\ ${ }^{a}$ Department of Farm Animal Health, Utrecht University, P.O. Box 80151, NL-3508 TD \\ Utrecht, The Netherlands \\ ${ }^{\mathrm{b}}$ Cryolab, Dijk 30, 5521 AZ Eersel, The Netherlands \\ ${ }^{\mathrm{c}}$ Section of Reproduction, Department of Production Animal Studies, Faculty of \\ Veterinary Science, University of Pretoria, Private Bag X04, Onderstepoort 0110, South \\ Africa \\ ${ }^{\mathrm{d}}$ Department Biochemistry and Cell Biology, Utrecht University, P.O. Box 80176, NL- \\ 3508 TD Utrecht, The Netherlands
}

\begin{abstract}
This study was done to determine the effects of processing techniques on the quality of semen from Dutch AI-bucks with the view on improving pregnancy rates after artificial insemination (AI) with liquid or frozen-thawed semen. Motility of spermatozoa was estimated under a microscope whereas the percentage live spermatozoa and the percentage live spermatozoa with intact acrosomes were determined by means of flow cytometry. Aspects of semen processing that were investigated are storage temperature of liquid semen (i), the effect of glycerol on liquid-stored semen (ii), removal of seminal plasma (iii) and type of extender (iv). The correlation between semen quality and fertility rates in inseminated does was also investigated.
\end{abstract}


The percentage motile spermatozoa in semen stored in liquid form for $72 \mathrm{~h}$ progressively declined over time, irrespective of whether storage occurred at 4 or $18{ }^{\circ} \mathrm{C}$. The percentage motile spermatozoa in semen stored at $18^{\circ} \mathrm{C}$ was similar to that in semen stored at $4{ }^{\circ} \mathrm{C}$ if stored for $24 \mathrm{~h}$ but lower if stored for $48 \mathrm{~h}$. Goats differ in the sensitivity of their spermatozoa to the deleterious effects of glycerol. Neither the removal of seminal plasma nor the type of extender had any effect on semen quality before freezing but semen frozen in a Tris-citric acid-glucose (TCG) buffer with egg yolk without removal of the seminal plasma had better quality after thawing than semen frozen in another diluent or after removal of seminal plasma.

Remarkably no significant correlation between fertility and membrane integrity of spermatozoa could be found. Thus, although integrity assays for spermatozoa are useful to asses resistance to semen handling, the validity of these assays for predicting fertility is questioned.

\section{Article Outline}

1. Introduction

2. Materials and methods

2.1. Extenders

2.2. Experimental design

2.3. Semen collection and initial evaluation

2.4. Preparation of samples for storage at 18,4 or at $-196^{\circ} \mathrm{C}$

2.5. Flow cytometry analysis

2.6. Measurement of fertility and correlation with semen quality

2.7. Statistical analysis

3. Results

3.1. Initial evaluation

3.2. Effects of liquid storage

3.2.1. Storage temperature

3.2.2. Glycerol in the extender

3.2.3. Effects of removal of seminal plasma and (iv) type of extender 
3.3. Correlation between results of different evaluation methods for buck semen

3.4. Correlation between semen quality and fertility

4. Discussion

Acknowledgements

References

\section{Introduction}

Artificial insemination (AI) holds several genetic and economic advantages for goat production. AI is the preferred method of introducing superior genes from individuals free of specific diseases and thereby improving the production of offspring. AI is also useful for breeding goats outside of their natural breeding, which runs from September to February in Western Europe, after oestrus has been induced and synchronised by manipulation of the photoperiod or the use of hormones. Such out of season breeding permits the production of adequate quantities of milk and equalises the need for labour throughout the year. Semen used for out of season breeding can be frozen-thawed or liquid-stored if collected from bucks held under artificial light in order to simulate the short days of the natural breeding season. Although higher pregnancy rates may be obtained with trans-abdominal insemination than with cervical insemination the former method may be prohibited due to ethical considerations [1]. Currently, the use of frozenthawed or liquid semen in goats in which oestrus was synchronised by means of hormone therapy results in pregnancy rates of approximately 61 or $64 \%$, respectively [16].

The extender used is a key factor in semen processing. Enzymes in the seminal plasma of bucks may interact with specific components of skimmed milk or egg yolk, rendering extenders containing these substances harmful to the bucks' spermatozoa [2], [16], [20] and [21]. A practical solution to this is to remove seminal plasma prior to admission of egg yolk or skimmed milk extenders to the spermatozoa. Despite of this possibility, in practise routinely extenders containing skimmed milk or egg yolk are directly added to undiluted semen [2], [5], [15], [16], [17] and [19]. We may note that seminal plasma also contains factors favourable for sperm survival, it is envisaged that specific inhibitors of the afore-mentioned enzymes could be added to extenders [20] and [21]. 
Storage temperature is another important factor in semen processing. Liquid goat semen destined for use within $12 \mathrm{~h}$ should be stored at $4{ }^{\circ} \mathrm{C}$ [15]. Significant temperature changes during storage of liquid semen are considered detrimental to semen quality and, under practical conditions, fluctuations in temperature may be easier to avoid at higher storage temperatures than at $4{ }^{\circ} \mathrm{C}$.

Glycerol is a cryoprotectant that is added to extenders used for freezing of spermatozoa. Glycerol reduces the viability of spermatozoa from humans and rams [8] and [9] but its effects on buck semen are unclear.

Better methods to dilute, wash and store semen may improve the results obtained with AI in goats. Several procedures to process buck semen were therefore evaluated in the present study. The quality of an ejaculate is often expressed as the number, motility and morphology of spermatozoa therein. These indicators of semen quality only have limited value for predicting the potential of a given insemination dose to fertilize the oocytes of a goat and result in a pregnancy. It is therefore important to use adequate evaluation techniques to compare different protocols for the processing of semen [12]. The percentage of motile spermatozoa in a sample is most commonly used to evaluate semen quality. Motility is usually determined subjectively and we therefore decided to compare the percentage of motile spermatozoa with viability and acrosomal integrity as objectively assessed by means of flow cytometry [12].

The aim of the current study was to modify existing protocols in order to improve the preservation of buck semen in liquid or frozen form. More specifically, the aims were to compare the effects of two extenders and the removing or not of seminal plasma on the quality of buck semen before and after freezing-thawing process, to compare the effects of two storing temperatures on the quality of cooled spermatozoa and, finally, to determine the effect of glycerol on the quality of spermatozoa kept at $18{ }^{\circ} \mathrm{C}$. Apart from the effects of the above treatments on semen quality, the correlation between semen quality and fertility of frozen-thawed spermatozoa was determined. 


\section{Materials and methods}

\subsection{Extenders}

The ejaculates were diluted in a Tris-citric acid-glucose (TCG) buffer or in a glucose solution, both at $37^{\circ} \mathrm{C}$. The TCG buffer contained $375 \mathrm{mM}$

tris(hydroxymethyl)aminomethane, $124 \mathrm{mM}$ citric acid, $41 \mathrm{mM}$ glucose, $375 \mathrm{mM}$ trehalose, $0.5 \mathrm{mg} / \mathrm{ml}$ dihydrostreptomycin and $0.3 \mathrm{mg} / \mathrm{ml}$ benzyl penicillin. The TCG buffer was prepared either with or without $20 \%(\mathrm{v} / \mathrm{v})$ egg yolk and $4 \%(\mathrm{v} / \mathrm{v})$ glycerol as described before [3]. The glucose solution buffer contained, $50 \mathrm{mM}$ d-glucose, $0.3 \mathrm{mg} / \mathrm{ml}$ benzyl penicillin, $0.5 \mathrm{mg} / \mathrm{ml}$ dihydrostreptomycin and was prepared either with or without $100 \mathrm{mg} / \mathrm{ml}$ skimmed milk and 4\% (v/v) glycerol. All solutions were adjusted to a $\mathrm{pH}$ of 7.0 and an osmolarity of 375 mOsm.

\subsection{Experimental design}

Each ejaculate was split into five aliquots immediately after collection and initial evaluation. Aliquot 1 was diluted in TCG buffer, centrifuged at $1200 \times g$ for $15 \mathrm{~min}$ and the supernatant containing the seminal plasma discarded. The dilution and centrifugation of aliquot 1 was repeated and the sediment then diluted in a TCG buffer with egg yolk and glycerol, before it was cooled to $4{ }^{\circ} \mathrm{C}$ and frozen. Aliquot 2 was diluted in the TCG buffer with egg yolk and glycerol and thereafter cooled to $4{ }^{\circ} \mathrm{C}$ and frozen. Aliquot 3 was treated in the same way as aliquot 1, except that a glucose solution was used prior to centrifugation and a glucose solution with skim milk and glycerol to dilute the sediment. Aliquot 4 was diluted in a glucose solution with skimmed milk and glycerol subsequent to which part thereof was cooled to $4{ }^{\circ} \mathrm{C}$ and frozen and part kept at $18{ }^{\circ} \mathrm{C}$ for $24 \mathrm{~h}$ (aliquot $4_{18}$ ). The fifth aliquot of each ejaculate was diluted in a glucose solution with skimmed milk and then divided into two aliquots of which one was cooled to $4{ }^{\circ} \mathrm{C}$ and kept at that temperature for $72 \mathrm{~h}$ (aliquot $5_{4}$ ), whereas the other was cooled to $18^{\circ} \mathrm{C}$ and kept at that temperature for $72 \mathrm{~h}$ (aliquot $5_{18}$ ). Section 2.1 provides the composition of each diluent. Fig. 1 shows how an ejaculate was split and the aliquots assigned to the different treatments. 


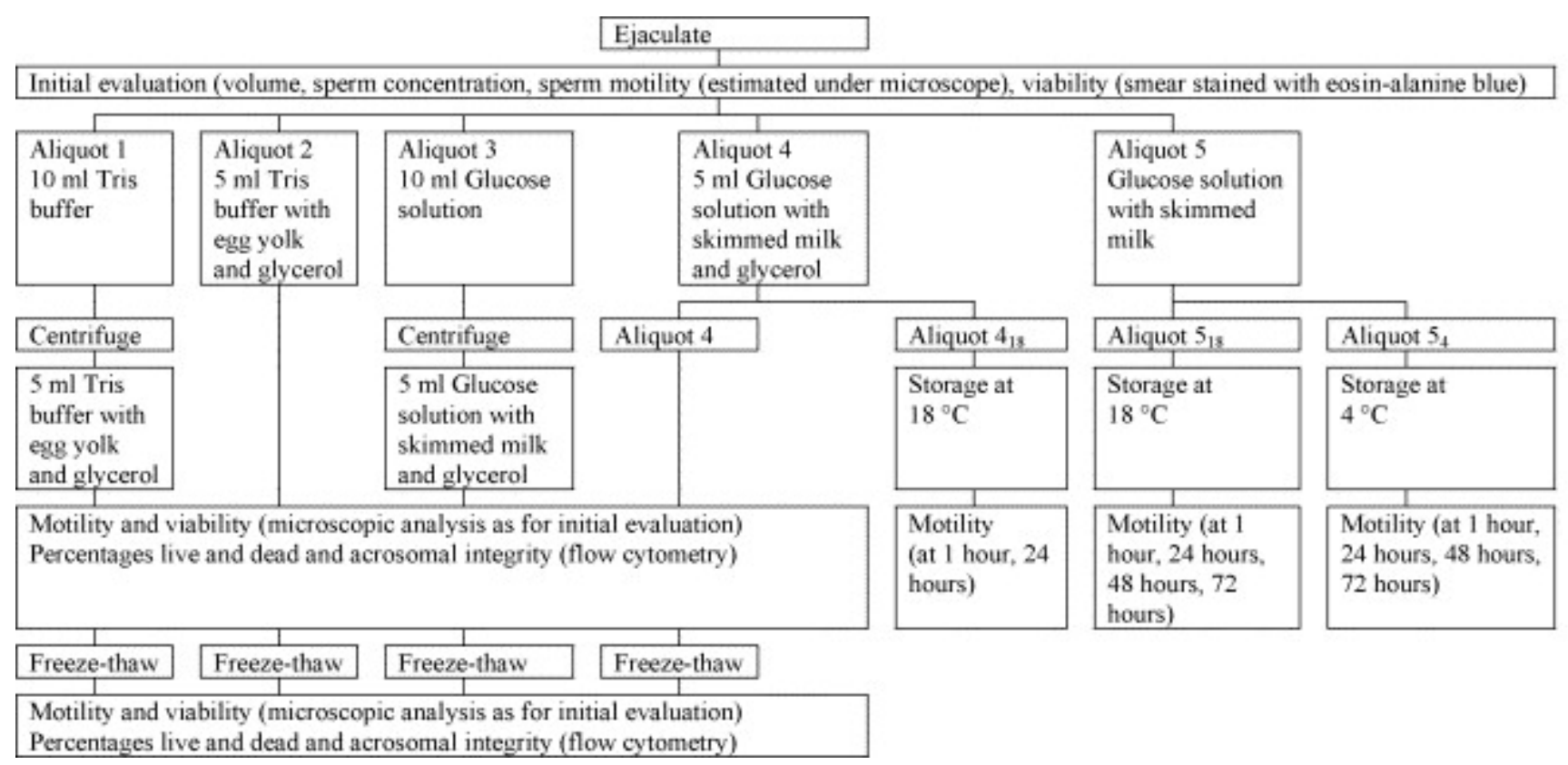

Fig. 1. Overview of the experimental design and allocation of semen to the different treatments (split sample, cross-over design). The objectives are to evaluate the influence of seminal plasma, determine the optimal extender in liquid en frozen-thawed semen, to evaluate the effect of the cryoprotectant glycerol, and the survival of spermatozoa in liquid semen at different temperatures over time.

Initially, the volume of an ejaculate, as well as the concentration, motility and viability of its spermatozoa were determined. Aliquots $1-4$ were evaluated for $\%$ of motile, $\%$ of viable, $\%$ live/dead and $\%$ of acrosome-intact spermatozoa before freezing and after thawing. The motility of aliquot $4{ }_{18}$ was determined after 1 and $24 \mathrm{~h}$ whereas the motility of aliquots $5_{4}$ and $5_{18}$ was determined after 1, 24, 48 and $72 \mathrm{~h}$. Fig. 1 shows which evaluations were performed on each aliquot as well as when the evaluations were done. All experiments were performed twice on two ejaculates that were separately collected at different times in a group of eight bucks.

\subsection{Semen collection and initial evaluation}

Eight Saanen bucks were selected on production and conformation characteristics by the Dutch AI Cooperation (Geiten KI Nederland; GKN). The bucks were individually housed in climate-controlled stables $\left(18^{\circ} \mathrm{C}\right)$. The photoperiod was $8 \mathrm{~h}$ of light and $16 \mathrm{~h}$ of 
darkness to simulate short days. Semen was collected by ejaculation into an artificial vagina $\left(37^{\circ} \mathrm{C}\right)$ in the presence of a goat in oestrus. Upon collection, each ejaculate was weighed to determine its volume. Twenty-five microlitre of the ejaculates was diluted in $5 \mathrm{ml}$ Tris-buffer (containing $20 \mathrm{mM}$ Tris and $130 \mathrm{mM} \mathrm{NaCl}$; Merck, International BV, Amsterdam, the Netherlands) and mixed on a vortex. The concentration of spermatozoa in the ejaculate was determined by placing $2 \mathrm{ml}$ of the suspension in an ACCUCELL photometer (IVM Technologies, L'Aigle, France). Then the total number of cells in the ejaculate was calculated. In order to assess the motility of spermatozoa $10 \mu \mathrm{l}$ of semen was diluted in $1 \mathrm{ml}$ Tris-buffer, $10 \mu \mathrm{l}$ of this suspension transferred to a warm $\left(37^{\circ} \mathrm{C}\right)$ glass slide and covered with a glass coverslip (at the same temperature). The slide was then placed on the warm stage of a standard phase contrast microscope (Olympus BX40, Olympus optical Co., Ltd., Japan). Two persons each assessed at least 10 microscope fields at $\times 100$ or $\times 200$ magnification and, from these assessments, estimated the percentage motile spermatozoa. The viability of the spermatozoa was evaluated on a smear stained with eosine-aniline blue (eosine yellow from Merck Ltd., Darmstadt, Germany and aniline from Gurr BDH Chemicals Ltd., Poole, England), using a brightfield illumination microscope (Olympus BX40, Olympus Optical Co., Ltd., Japan) at $\times 1000$ magnification.

\subsection{Preparation of samples for storage at 18,4 or at $-196{ }^{\circ} \mathrm{C}$}

After collection the semen was diluted in the TCG buffer at $37^{\circ} \mathrm{C}$ or in the glucose solution, which was also at $37^{\circ} \mathrm{C}$, to a final concentration of $400 \times 10^{6}$ spermatozoa/ml. After dilution the semen was stored in cotton-isolated containers in a Styropor box and subsequently cooled to room temperature over $2 \mathrm{~h}$. Thereafter, the tubes were either stored in a dry and dark container at $18{ }^{\circ} \mathrm{C}$ or cooled and equilibrated over a period of $2 \mathrm{~h}$ in a refrigerator at $4{ }^{\circ} \mathrm{C}$.

Aliquots $4_{18}$ and $5_{18}$ were stored at $18{ }^{\circ} \mathrm{C}$ for $24 \mathrm{~h}$ and $72 \mathrm{~h}$, respectively, whereas aliquot 54 was stored at $4{ }^{\circ} \mathrm{C}$ for $72 \mathrm{~h}$.

Before cooling and equilibration the spermatozoa destined for cryopreservation (aliquots 1-4) was further diluted in their accessory extender to a final concentration of $400 \times 10^{6}$ spermatozoa/ $\mathrm{ml}$. Once cooled and equilibrated at $4{ }^{\circ} \mathrm{C}$ these aliquots were 
packaged in pre-printed $0.5 \mathrm{ml}$ French straws, using a machine that automatically fills and seals the straws (IMV, Cedex, France). The straws were then frozen in a Kryo 10 series II freezer (Planer Products Ltd., Sunbury-on-Thames, UK) according to an automated freezing protocol. The cooling rate from 4 to $-8{ }^{\circ} \mathrm{C}$ was $35^{\circ} \mathrm{C} / \mathrm{min}$, and thereafter $30^{\circ} \mathrm{C} / \mathrm{min}$ to $-140{ }^{\circ} \mathrm{C}$. Finally, the straws were stored in liquid nitrogen. In each experiment straws were thawed in a water bath at $37^{\circ} \mathrm{C}$ for $1 \mathrm{~min}$.

\subsection{Flow cytometry analysis}

The semen samples were diluted 1:10 in a solution containing phosphate buffered saline (PBS; $1.54 \mathrm{mM} \mathrm{KH}_{2} \mathrm{PO}_{4}, 155.17 \mathrm{mM} \mathrm{NaCl}, 2.71 \mathrm{mM} \mathrm{Na}_{2} \mathrm{HPO}_{4}$; pH 7.2, Gibco, UK) to which was added $0.1 \%(w / v)$ polyvinyl alcohol (PVA; Sigma, St. Louis, USA). In order to distinguish live and dead spermatozoa they were stained with SYBR-14 ${ }^{\circledR}$ dye and propidium iodide (PI) that were both present in the LIVE/DEAD staining kit from Molecular Probes, Inc. (Eugene, OR, USA) and used according to the supplier's protocol. For membrane integrity assessments $1 \mathrm{ml}$ of semen samples that were diluted 1:100 in PBS with $0.1 \%$ (w/v) PVA were stained for $10 \mathrm{~min}$ at $37{ }^{\circ} \mathrm{C}$ with $50 \mathrm{ng} / \mathrm{ml}$ fluorescein isothiocyanate-conjugated peanut agglutinin (FITC-PNA; EY Laboratories, Inc., San Mateo, LA, USA) and $0.4 \mu \mathrm{g} / \mathrm{ml}$ PI (Sigma Chemical Company, St. Louis, USA). The cells $\left(40 \times 10^{6} / \mathrm{ml}\right.$ for SYBR-14 ${ }^{\circledR} / \mathrm{PI}$ and $4 \times 10^{6} / \mathrm{ml}$ for FITC-PNA/PI) were then analysed for green and red fluorescence by flow cytometry using a FACScan equipped with an argon laser (488 nm, excitation line, Becton Dickinson, San Jose, CA). Semen specific events were gated for further analysis on the basis of their specific forward and side ways scatter properties [10] and [11].

The fluorescence detectors (FL $1=500-530 \mathrm{~nm}$ and FL $3>605 \mathrm{~nm}$ ) were set to asses the amount of the red and green emission of the sperm subpopulations in order to analyse plasma membrane- and acrosome-integrity according to previously described methodology [18]. Liquid semen samples were evaluated within 6-8 h after collection and within $1 \mathrm{~h}$ post-thawing for frozen samples. 


\subsection{Measurement of fertility and correlation with semen quality}

At least 15 ejaculates were obtained from each of 6 bucks, distributed to a minimum of 8 and a maximum of 16 different farms, in order to evaluate fertility. Ejaculates were diluted to a concentration of $400 \times 10^{6}$ spermatozoa per $\mathrm{ml}$ in a glucose solution with skimmed milk (as described above for aliquot 5), cooled to $4{ }^{\circ} \mathrm{C}$ and kept at that temperature for a maximum of $8 \mathrm{~h}$ after ejaculation before the semen was used to inseminate does.

The oestrous cycles of 1178 goats (70-260 per buck) were synchronised with progestagen-impregnated intravaginal sponges (Chrono-gest, Intervet, Boxmeer, The Netherlands) for 11 days. On day 9, the does were treated intramuscularly with 400$600 \mathrm{IU}$ of ECG (Folligonan, Intervet, Boxmeer, The Netherlands) and $2 \mathrm{ml}$ of PGF2 $\alpha$. Using $0.5 \mathrm{ml}$ of liquid semen containing $200 \times 10^{6}$ spermatozoa, an experienced inseminator performed a single intracervical insemination on each doe $41-45 \mathrm{~h}$ after sponge removal [16]. The does' hind legs were lifted off the ground so that the animal was standing on the forelegs. The hind legs were subsequently folded under the body and the body was raised in a near vertical position during insemination.

The techniques described in Sections Sections 2.3 and 2.5 were used to determine the percentages progressively motile, live and live acrosome-intact spermatozoa in the ejaculates. These variables of semen quality were correlated with the kidding rate of does obtained after AI with liquid semen within $8 \mathrm{~h}$ of collection.

\subsection{Statistical analysis}

The parameters of fresh semen quality are presented as means \pm S.D. The flow cytometric data were analysed with Cell Quest software (Becton Dickinson) and WinMDI (Windows Multiple Document Interface for Flow Cytometry, Version 2.8; http://facs.scripps.edu/software.html). Region and quadrant analyses were done on 10,000-gated spermatozoa from each sample. Further analyses of the scores were done with Excel (Microsoft) and SPSS (SPSS Inc., USA; Version 10.0). For the analysis of storage temperature, data of eight bucks were included and a paired sample $t$-test was used to compare the mean $\%$ of motile spermatozoa at different time periods. A one-way ANOVA was used to establish the difference between the two storage temperatures at 24, 
48 and $72 \mathrm{~h}$. The effects of storage temperature and glycerol on motility during liquid storage were respectively determined by means of a one-way ANOVA whereas multiple comparisons of means were done with Bonferroni's test. Different combination of effect of removal of seminal plasma in combination with type of extender $(n=4)$ was determined by means of an one-way ANOVA with a Bonferroni post hoc test. Initially, a square root transformation was performed to normalise the data on the percentage of live spermatozoa (SYBR-14 ${ }^{\circledR} / \mathrm{PI}$ ). The separate values for fertility of individual bucks were scored as the number of goats that produced kids divided by the number of goats that received semen from a particular buck through AI. Subsequently, the correlation coefficient between fertility and the percentages progressively motile, live and live acrosome-intact spermatozoa were assessed.

\section{Results}

\subsection{Initial evaluation}

The volume of the ejaculates was $1.3 \pm 0.5 \mathrm{ml}$, and the concentration of spermatozoa was $6.2 \pm 3.9 \times 10^{9}$ per ml. Thus, the number of spermatozoa per ejaculate was $7.6 \pm 5.4 \times 10^{9}$. Ejaculates had $76 \pm 11 \%$ motile, and $92 \pm 3 \%$ viable spermatozoa.

\subsection{Effects of liquid storage}

\subsubsection{Storage temperature}

Fig. 2 shows the effect of storage temperature over time. There was a significant decline in the percentage of motile spermatozoa during the first $24 \mathrm{~h}$ of storage $(p<0.005$ for spermatozoa stored at $18{ }^{\circ} \mathrm{C}$ and $p<0.01$ for spermatozoa stored at $4{ }^{\circ} \mathrm{C}, p<0.01$ ), although there was no significant difference in motility of spermatozoa between the temperature groups $(p>0.05)$. Storage from 24 to $48 \mathrm{~h}$ resulted in a prominent decline $\left(18^{\circ} \mathrm{C}, p<0.001 ; 4^{\circ} \mathrm{C}, p<0.005\right)$ in the percentage of motile spermatozoa. By $48 \mathrm{~h}$, the percentage motile spermatozoa was significantly lower in the group stored at $18^{\circ} \mathrm{C}$ than in the group stored at $4{ }^{\circ} \mathrm{C}(p<0.001)$. The percentage of motile spermatozoa further decreased during storage between 48 and $72 \mathrm{~h}\left(18{ }^{\circ} \mathrm{C}, p<0.05 ; 4{ }^{\circ} \mathrm{C}, p<0.05\right)$ but there was no significant difference in motility of spermatozoa between the temperature groups $(p>0.05)$. 


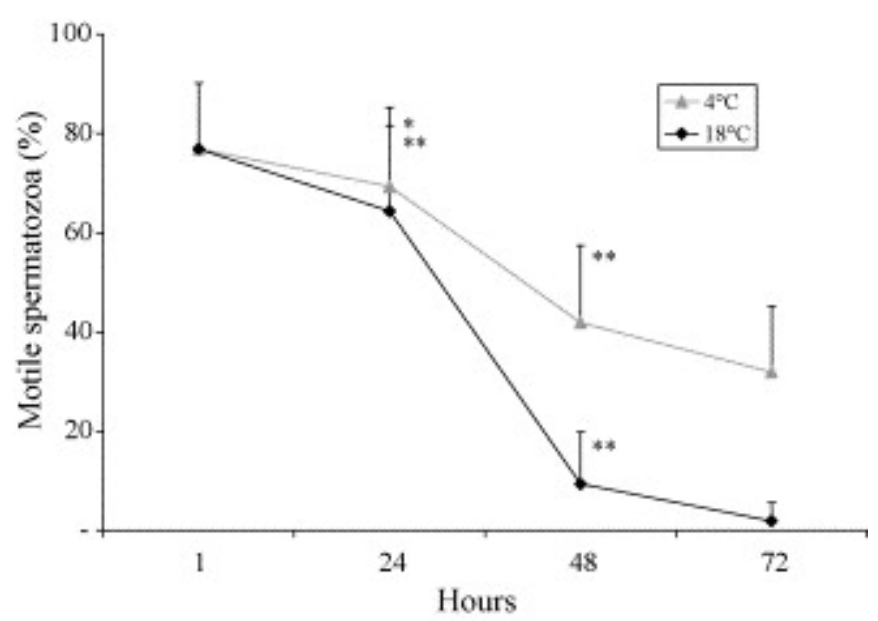

Fig. 2. Percentage (mean \pm S.D.) of motile spermatozoa in the ejaculates of eight bucks stored for up to $72 \mathrm{~h}$ at 18 and $4{ }^{\circ} \mathrm{C}$ in a glucose solution containing skimmed milk.

\subsubsection{Glycerol in the extender}

Glycerol had a negative effect on the motility of spermatozoa over a period of $24 \mathrm{~h}$ and the effect varied among bucks (Fig. 3). The spermatozoa of one buck were intolerant towards storage in a glucose solution with skimmed milk and glycerol, as all spermatozoa were immotile within $1 \mathrm{~h}$. For three other bucks motility of spermatozoa had completely vanished within $24 \mathrm{~h}$ of storage. For the remaining three bucks glycerol did not affect motility of spermatozoa during storage at $18{ }^{\circ} \mathrm{C}$ (Fig. 3).

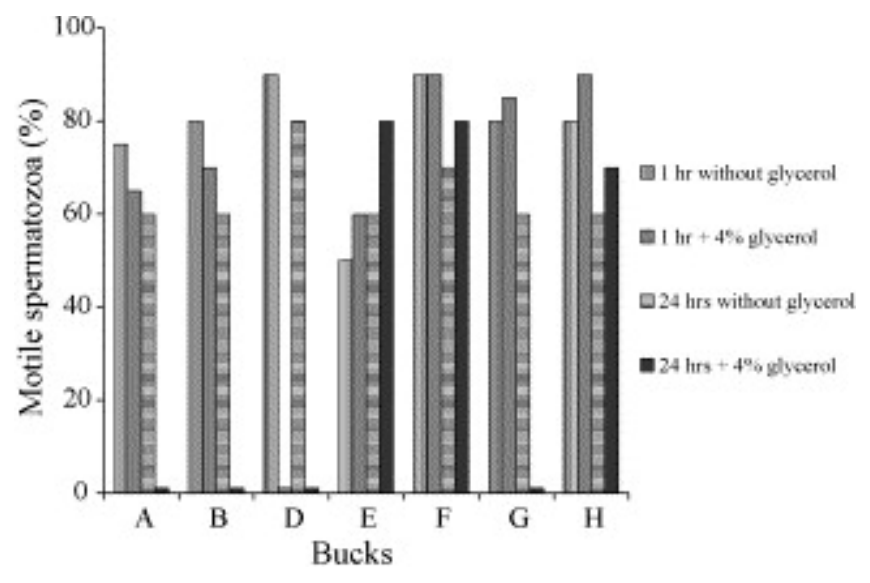


Fig. 3. Effect of glycerol on the percentages of motile spermatozoa of seven bucks (samples were stored for 1 and $24 \mathrm{~h}$ at $18^{\circ} \mathrm{C}$ in a glucose solution containing skimmed milk with or without $4 \%$ glycerol).

\subsubsection{Effects of removal of seminal plasma and (iv) type of extender}

Fig. 4 shows that the removal of seminal plasma had no effect on the percentage of live spermatozoa before freezing ( $p=0.2$, both buffers) or after thawing ( $p=0.6$, glucose buffer only). However, in TCG buffer removal of seminal plasma resulted in a significant higher proportion of deteriorated spermatozoa compared to TCG buffer with seminal plasma. Irrespective to the presence of seminal plasma TCG buffer was superior to the Glucose buffer when considering cryoresistance of the spermatozoa. This is best illustrated in Fig. 4 that shows that TCG buffer with seminal plasma did only marginally cause cryodamage to the spermatozoa. Furthermore, the survival before freezing was similar in TCG buffer with egg yolk or glucose solution containing skimmed milk $(p=0.6)$. Fig. 4 shows that a significantly higher percentage of spermatozoa diluted in TCG buffer with egg yolk without washing survived the freeze-thaw process compared to spermatozoa exposed to the other three treatments $(p<0.005)$.

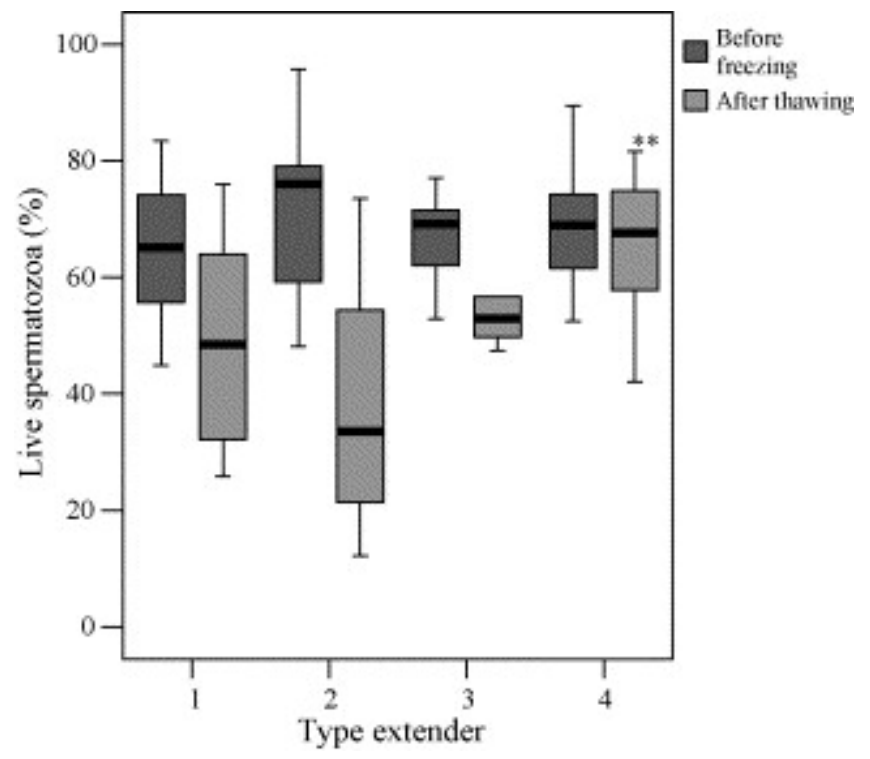

Fig. 4. Effects of two diluents (glucose solution with skimmed milk (1 and 2) and TCG with egg yolk (3 and 4 ) and the removal (1 and 3) or not (2 and 4) of seminal plasma on the percentage live spermatozoa (flow cytometry after SYBR-14 ${ }^{\circledR} /$ PI staining) before 
freezing or after thawing (horizontal bars indicate the median, shaded blocks the interquartile range that contains $50 \%$ of values and the vertical lines the highest and lowest values after exclusion of outliers and extremes).

\subsection{Correlation between results of different evaluation methods for buck semen}

Fig. 5a shows that the proportion of cells with intact membranes correlated with the proportion of motile cells, both, for spermatozoa stored at 18 and at $4{ }^{\circ} \mathrm{C}(R=0.77$ and 0.98 , respectively). Similarly, Fig. $5 \mathrm{~b}$ shows that the percentage of motile spermatozoa correlated with the percentage of live spermatozoa with intact acrosomes $(R=0.94$ for spermatozoa stored at $18{ }^{\circ} \mathrm{C}$ and $R=0.93$ for spermatozoa stored at $4{ }^{\circ} \mathrm{C}$ ).
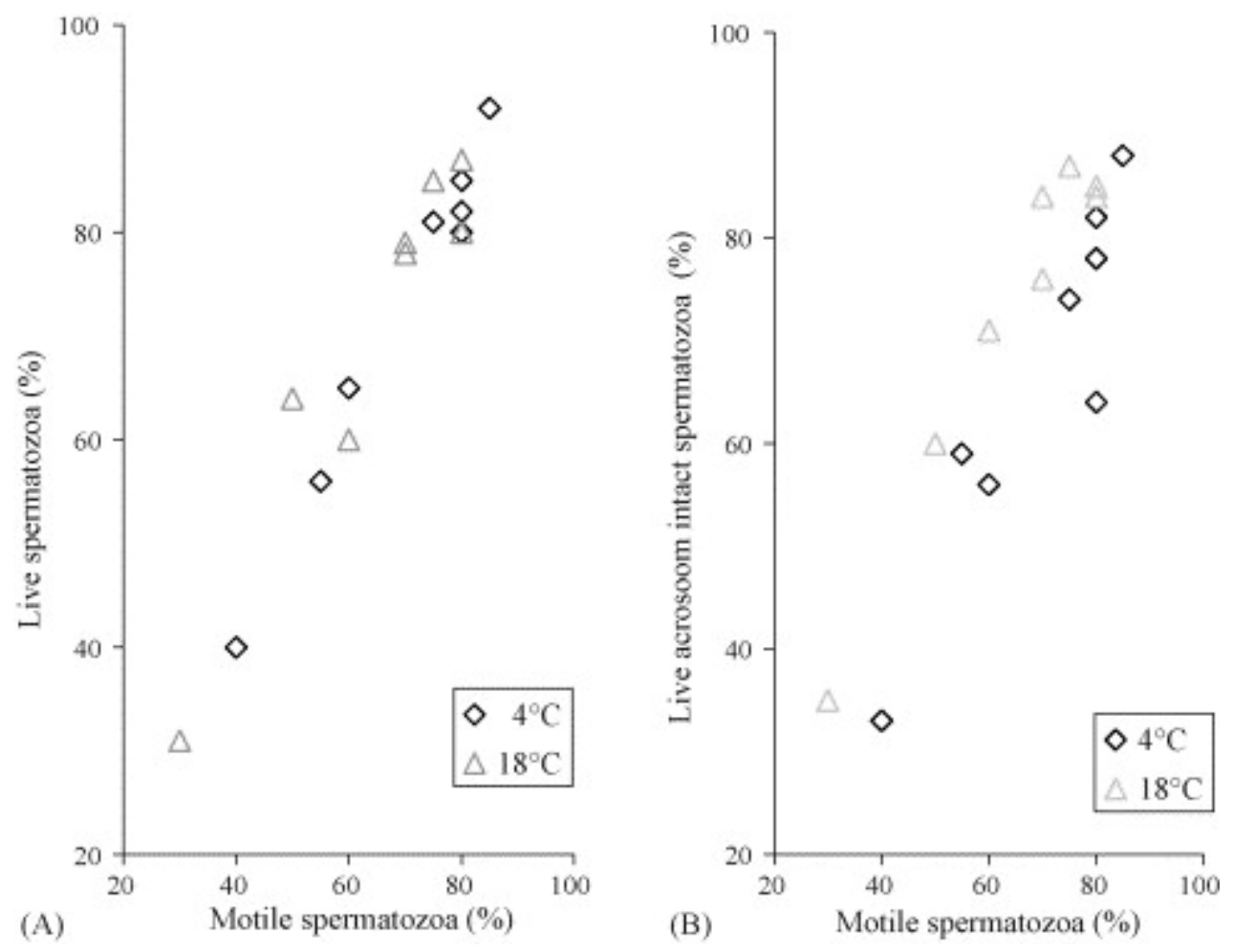

Fig. 5. Scatter plots between the percentage of motile spermatozoa as estimated under a microscope and the percentage of live spermatozoa as determined by means of flow cytometry (A) SYBR-14 ${ }^{\circledR /}$ PI staining (B) and the percentage of live spermatozoa with intact acrosomes as determined by means of flow cytometry after PNA-FITC-PI staining (spermatozoa from eight bucks stored for $24 \mathrm{~h}$ at 4 or $18^{\circ} \mathrm{C}$ ). 


\subsection{Correlation between semen quality and fertility}

The mean kidding rates after insemination with liquid semen varied from 49 to $73 \%$ between the individual bucks. Since 70 up to 208 does were inseminated with semen from one buck, more than one ejaculate had to be used from each buck, with the result that the fertility percentages of a buck varied by $7-10 \%$ among ejaculates. Kidding rates obtained after AI correlated poorly with the percentage motile cells in the ejaculates used for the inseminations, irrespective of whether motility was assessed after storage at 18 or at $4{ }^{\circ} \mathrm{C}\left(R=0.36\right.$ for semen from six bucks stored at $18{ }^{\circ} \mathrm{C}$ and $R=0.35$ for semen from the same six bucks stored at $4{ }^{\circ} \mathrm{C}$, Fig. 6a). The percentage of live spermatozoa and the mean kidding rates showed stronger correlations $\left(R=0.44\right.$ at $18{ }^{\circ} \mathrm{C}$ and $R=0.27$ at $4{ }^{\circ} \mathrm{C}$, Fig. 6b). Fig. 6c shows that the percentage of live spermatozoa with intact acrosomes correlated poorly with fertility $\left(R=0.18\right.$ at $18{ }^{\circ} \mathrm{C}$ and $R=0.27$ at $\left.4{ }^{\circ} \mathrm{C}\right)$.

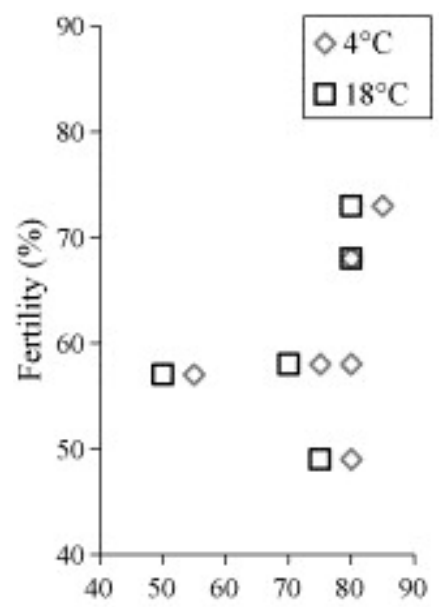

(A) Motile spermatozoa (\%)

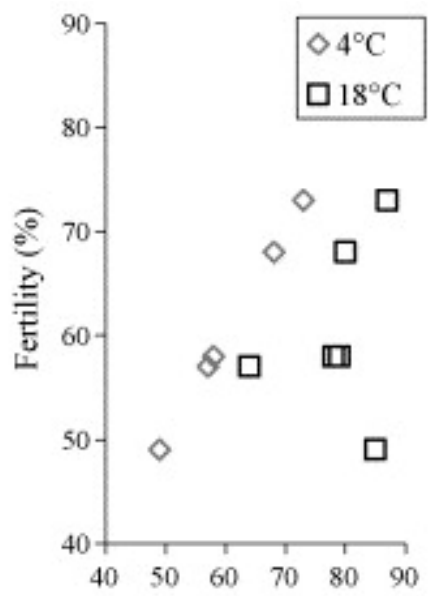

(B) Live spermatozoa (\%)

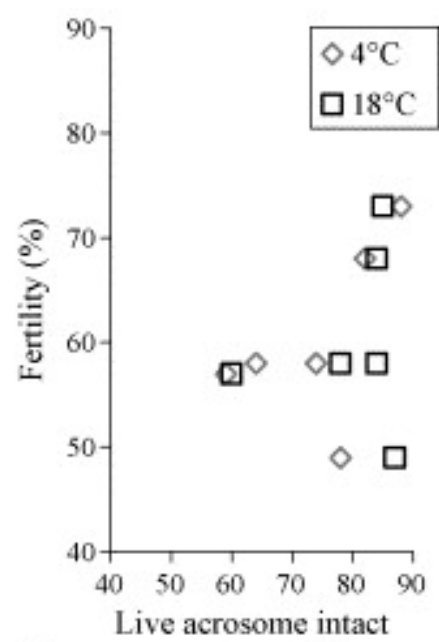

(C) spermatozoa (\%)

Fig. 6. Correlation between fertility (percentage of inseminated does that produced kids) and the (A) percentages of motile spermatozoa estimated under a microscope; (B) live spermatozoa determined by flow cytometry after staining with SYBR-14 ${ }^{\circledR} / \mathrm{PI}$; (C) live spermatozoa with intact acrosomes determined by flow cytometry after staining with PNA-FITC/PI. Sperm from six bucks were stored for $24 \mathrm{~h}$ at 4 or $18{ }^{\circ} \mathrm{C}$. 


\section{Discussion}

This study shows that storing goat semen for $24 \mathrm{~h}$ at $18{ }^{\circ} \mathrm{C}$ results in similar motility of spermatozoa than storing such semen for $24 \mathrm{~h}$ at $4{ }^{\circ} \mathrm{C}$. Practically this outcome implies that liquid semen does not have to be cooled to $4{ }^{\circ} \mathrm{C}$, nor stored and transported under refrigerator conditions when used within a day after collection.

Although it is known that glycerol can have a negative effect on semen quality, the number of bucks that reacted negatively on the glycerol was impressive. Glycerol, despite its value as cryoprotectant, is noxious to membrane integrity of spermatozoa of humans and sheep [9] and [10], and also metabolically toxic to these cells [25]. Glycerol is the preferred cryoprotectant for goat semen because others are less effective [25]. Glycerol increases osmotic stress and differences among individuals in their sensitivity to glycerol may therefore be due to different abilities to modulate the passage of water and other molecules through cell membranes. Perhaps bucks with a limited resistance to glycerol and/or extender(s) should be eliminated from AI services, since their frozen semen will not result in successful AI.

Even though flow cytometric analysis is considered more accurate to assess semen quality and preservation methods then conventional microscopic evaluation techniques [10], [11] and [18], we found a very high correlation between the results of flow cytometric analysis and microscopical assessment of motility (Fig. 5a and b). The percentages of live acrosome-intact (FITC-PNA/PI), and live spermatozoa (SYBR$\left.14^{\circledR} / \mathrm{PI}\right)$ were consistently similar as could be expected. Unlike earlier studies suggesting that semen analysis can partly predict fertility of bucks [5] and [19] the current study shows no significant correlations between the percentages of motile spermatozoa and live spermatozoa with intact acrosomes, respectively, and fertility. The correlation between the percentage live spermatozoa and fertility is somewhat stronger and more experiments should be performed to assess the reliability of this outcome. Flow cytometry allows the objective, rapid and simultaneous analysis of a number of properties in a large number of spermatozoa [4], suggesting that the results of flow cytometric analysis may allow the estimation of the fertility of a semen sample. Yet, it has been shown that combining the results of various sperm function tests does not result in more reliable estimation of fertility [4]. Microscopic evaluation of motility is subjective, as it entirely depends on the 
ability and experience of an operator estimating motility under a phase-contrast microscope. Even so, the microscopic assessment of motility after thawing or immediately prior to insemination is simple, easy and quick and provides the parameter of choice under field conditions to indicate the degree of damage inflicted by cryopreservation. This study shows that motility assessments performed by the experienced operator reliably corresponded to flow cytometric viability assessments. This may implement that the purchase of expensive flow cytometric equipment (for accurate integrity assessments of spermatozoa) is not required to improve goat AI.

The current study shows that the removal of the seminal plasma does not affect the semen quality of liquid-stored or frozen-thawed semen in Dutch dairy goat bucks. Buck seminal plasma contains two compounds that can react with ingredients of diluents. Bulbourethral secreting glycoprotein-60 (BUSgp60) has a triacylglycerol hydrolase activity that causes deterioration of buck spermatozoa in skimmed milk extender. BUSgp60 causes a decrease in the percentage of motile spermatozoa, a deterioration in the quality of movement, the breakage of acrosomes, and the death of buck spermatozoa [16], [20] and [21]. The other compound is egg yolk coagulating enzyme (EYCE) which has phospolipase A2 activity and therefore hydrolyses egg yolk phosphatidylcholine (PC) into fatty acid and lysophosphatidylcholine (LPC) [2] and [5]. LPC acts like a detergent on biomembranes and is therefore toxic to buck spermatozoa. However, the strength of the detergent properties of LPC depend on the amount formed, temperature (enzyme activity and thus LPC formation is temperature dependent), dilution or degree of removal of seminal plasma, season of semen production and breed of fowl providing the egg yolk [16]. In the buck, removal of seminal plasma by washing the spermatozoa immediately after collection increases the percentage of live cells and their motility during storage in egg yolk or milk diluents [6] and [22] although this effect does not always occur [7]. The storability of ejaculated and washed semen is less than that of epididymal semen [5], [14] and [19], suggesting that seminal plasma contains compounds detrimental to in vitro storage.

The current study shows that washing of semen of Dutch dairy goat bucks does not affect their viability before freezing or after thawing, implying that under field conditions washing is not necessary in The Netherlands. In other breeds washing can be necessarily 
[13] and [23]. Since the type of season affects the composition of seminal plasma this experiment was performed during the natural breeding period in order to obtain optimal results. Trehalose, present in the TCG-buffered extender used in the current study, has been shown to reduce acrosomal detoriation [26]. In the current study the type of extender had no effect on semen quality before freezing because it reduces deterioration of spermatozoa compared to the other extenders used. In contrast, the current study shows that the extender containing TCG and egg yolk is preferred for freezing buck spermatozoa because it reduces live acrosome-intact spermatozoa less than the other extenders used. This is partly consistent with the findings of an earlier study [2] in which it was shown that a TCG diluent containing egg yolk reduced deterioration of spermatozoa as assessed with motility characteristics compared to other extenders. In contrast, in the same study an increase was noted of acrosomal damage. Egg yolk has the property to alleviate cold shock by its ability to shield membranes. Although it is well known in farm animals that egg yolk protects sperm cell membranes, egg yolk may also destabilize such membranes, as shown by an increased frequency of acrosomal damage associated with an increase in the concentration of egg yolk [24]. The bucks we used showed large differences in the quality of their semen with respect to motility, percentage of live cells and percentage of intact acrosomes before freezing and after thawing. Although the bucks performed consistently, it is arguable whether or not to draw strong conclusions from mean values.

In conclusion, semen analyses are useful to determine cryoresistance, reaction of spermatozoa to extender and effect of removal of seminal plasma, although they may not predict actual fertilizing potential, but they may predict low fertility and allow exclusion of those samples or even males from an artificial insemination program [12]. It is therefore advisable to test each buck in order to determine the preferred treatment of its semen with respect to resistance to glycerol, extender and removal of seminal plasma. Based on these tests, the most preferred storage treatment can be adapted for goat AI. 


\section{References}

[1] Gezondheids-en Welzijnswet voor Dieren. http://wettenoverheidnl.

[2] E.M. Aboagla and T. Terada, Effects of egg yolk during the freezing step of cryopreservation on the viability of goat spermatozoa, Theriogenology 62 (2004), pp. $1160-1172$.

[3] E.M. Aboagla and T. Terada, Trehalose-enhanced fluidity of the goat sperm membrane and its protection during freezing, Biol Reprod 69 (2003), pp. 1245-1250. [4] B. Colenbrander, B.M. Gadella and T.A. Stout, The predictive value of semen analysis in the evaluation of stallion fertility, Reprod Domest Anim 38 (2003), pp. 305311.

[5] J.M. Corteel, Effects of seminal plasma on the survival and fertility of spermatozoa kept in vitro, Reprod Nutr Dev 20 (1980), pp. 1111-1123.

[6] J.M. Corteel, Viabilité des spermatozoides de bouc conservés et congelés avec ou sans leur plasma seminal: effet du glucose, Ann Biol Anim, Biochim, Biophys 14 (1974) (4-B), pp. 741-745.

[7] H. Gacitua and A. Arav, Successful pregnancies with directional freezing of large volume buck semen, Theriogenology 63 (2005), pp. 931-938.

[8] D.Y. Gao, J. Liu, C. Liu, L.E. McGann, P.F. Watson and F.W. Kleinhans et al., Prevention of osmotic injury to human spermatozoa during addition and removal of glycerol, Hum Reprod 10 (1995), pp. 1109-1122.

[9] J. Gil, N. Lundeheim, L. Soderquist and H. Rodriiuez-Martinez, Influence of extender, temperature, and addition of glycerol on post-thaw sperm parameters in ram semen, Theriogenology 59 (2003), pp. 1241-1255.

[10] J.K. Graham, Assessment of sperm quality: a flow cytometric approach, Anim Reprod Sci 68 (2001), pp. 239-247.

[11] J.K. Graham, E. Kunze and R.H. Hammerstedt, Analysis of sperm cell viability, acrosomal integrity, and mitochondrial function using flow cytometry, Biol Reprod $\mathbf{4 3}$ (1990), pp. 55-64.

[12] J.K. Graham and E. Moce, Fertility evaluation of frozen/thawed semen, Theriogenology 64 (2005), pp. 492-504. 
[13] A. Iritani and Y. Nishikawa, Studies on the egg-yolk coagulating factors in goat semen. II. Properties of the coagulating factor and influential conditions for coagulation, Proceeding of Silver Jubilee Lab Animal Husbandry Kyoto University (1961), pp. $97-$ 104.

[14] C.N. Kundu, J. Chakraborty, P. Dutta, D. Bhattacharyya, A. Ghosh and G.C. Majumder, Development of a simple sperm cryopreservation model using a chemically defined medium and goat cauda epididymal spermatozoa, Cryobiology 40 (2000), pp. $117-125$.

[15] V.S. La Falci, H. Tortorella, J.L. Rodrigues and A. Brandelli, Seasonal variation of goat seminal plasma proteins, Theriogenology 57 (2002), pp. 1035-1048.

[16] B. Leboeuf, B. Restall and S. Salamon, Production and storage of goat semen for artificial insemination, Anim Reprod Sci 62 (2000), pp. 113-141.

[17] M.A. Memon, K.N. Bretzlaff and R.S. Ott, Effect of washing on motility and acrosome morphology of frozen-thawed goat spermatozoa, Am J Vet Res 46 (1985), pp. $473-475$.

[18] S. Nagy, J. Jansen, E.K. Topper and B.M. Gadella, A triple-stain flow cytometric method to assess plasma- and acrosome-membrane integrity of cryopreserved bovine sperm immediately after thawing in presence of egg-yolk particles, Biol Reprod $\mathbf{6 8}$ (2003), pp. 1828-1835.

[19] J.F. Nunes, J.M. Corteel, Y. Combarnous and G. Baril, Role of seminal plasma in the in vitro survival of goat sperm, Reprod Nutr Dev 22 (1982), pp. 611-620. [20] M.T. Pellicer-Rubio and Y. Combarnous, Deterioration of goat spermatozoa in skimmed milk-based extenders as a result of oleic acid released by the bulbourethral lipase BUSgp60, J Reprod Fertil 112 (1998), pp. 95-105.

[21] M.T. Pellicer-Rubio, T. Magallon and Y. Combarnous, Deterioration of goat sperm viability in milk extenders is due to a bulbourethral 60-kilodalton glycoprotein with triglyceride lipase activity, Biol Reprod 57 (1997), pp. 1023-1031.

[22] A.J. Ritar and S. Salamon, Effects of seminal plasma and of its removal and of egg yolk in the diluent on the survival of fresh and frozen-thawed spermatozoa of the Angora goat, Aust J Biol Sci 35 (1982), pp. 305-312. 
[23] A. Roy, Egg yolk-coagulating enzyme in the semen and Cowper's gland of the goat, Nature 179 (1957), pp. 318-319.

[24] R.L. Smith, W.E. Berndtson, M.B. Unal and B.W. Pickett, Influence of percent egg yolk during cooling and freezing on survival of bovine spermatozoa, J Dairy Sci 62 (1979), pp. 1297-1303.

[25] P.F. Watson, The causes of reduced fertility with cryopreserved semen, Anim Reprod Sci 60-61 (2000), pp. 481-492.

[26] C. Yildiz, A. Kaya, M. Aksoy and T. Tekeli, Influence of sugar supplementation of the extender on motility, viability and acrosomal integrity of dog spermatozoa during freezing, Theriogenology 54 (2000), pp. 579-585.

Corresponding author. Tel.: +31 30 2531248; fax: +31 302521887 . 